Research and Devel opment of Super conduct ing Magnet i c Separ at i on Syst em for Powder ed Pr oduct $\mathrm{s}$

\begin{tabular}{|l|l|}
\hline 著者 & $\begin{array}{l}\text { M SH MA F, TERADA T, AK YANA Y, I ZUM Y, } \\
\text { OKAZAK H, N SH JI MA S }\end{array}$ \\
\hline $\begin{array}{l}\text { j our nal or } \\
\text { publ i cat i on t i t l e }\end{array}$ & I EEE tr ansact i ons on appl i ed super conduct i vi ty \\
\hline vol une & 18 \\
\hline number & 2 \\
\hline page range & 824.827 \\
\hline year & 2008 . 06 \\
\hline URL & ht t p: //hdl . handl e. net /10098/2397 \\
\hline
\end{tabular}




\title{
Research and Development of Superconducting Magnetic Separation System for Powdered Products
}

\author{
F. Mishima, T. Terada, Y. Akiyama, Y. Izumi, H. Okazaki, and S. Nishijima
}

\begin{abstract}
The superconducting magnetic separation system which separates ferromagnetic particles from powder mixture has been developed. It is required to remove the impurities from the powdered products without using chemical or heating processing. The impurities are usually powdered stainless steel from the piping which are deformed strongly and shows ferromagnetism. Magnetic separation using permanent magnets have been used for the separation. The conventional magnetic separation system has not shown the expected performance especially for the small sized impurities. To solve the problem, superconducting magnetic separation system was developed. We used the magnet with magnetic field up to $2 \mathrm{~T}$. The boa size was $100 \mathrm{~mm}$ in diameter. Inside of the magnetic boa the magnetic filters are located to produce the high magnetic gradient. The performance of the separator was reported together with the design guideline.
\end{abstract}

Index Terms-Ferromagnetic materials, magnetic force, magnetic separation, superconducting magnet.

\section{INTRODUCTION}

$\mathbf{R}$ ECENTLY, interfusion of stainless powders in the product which causes the quality loss became acute problem in industrial factory such as the foods, the medicines and the chemicals, and so on. The stainless powder (10-100 $\mu \mathrm{m}$ of particle diameter) is usually generated from the moving parts of manufacturing process by abrasion. Since the chemical separation or heat-treatment increases the process cost, the separation as a particle is required. The stainless powder which was strongly deformed shows the ferromagnetism for the martensitic transformation. Therefore, a magnetic separation has been made for separating them as particles. In the magnetic separation of powder state without a liquid medium, the cohesive force between particles is strong compared with that in the wet type magnetic separation. For this reason, the blockades in a separator are often to be caused, and the separation efficiency is decreased. When the diameter of object for separation became smaller, the cohesive force increases because specific surface of particle becomes larger. The magnetic force to the object becomes smaller. This makes the magnetic separation more difficult. [1] The permanent magnet and electromagnet have been used for the separation of the powders. The neodymium magnet makes it possible to make the higher magnetic field of $1 \mathrm{~T}$. Though the neodymium magnet makes it possible to make

Manuscript received August 28, 2007.

F. Mishima, T. Terada, Y. Akiyama, Y. Izumi and S. Nishijima are with the Division of Sustainable Energy and Environmental Engineering, Osaka University, Osaka 565-0871, Japan (e-mail: f-mishima@qb.see. eng.osaka-u.ac.jp; t-terada@qb.see.eng.osaka-u.ac.jp; yoko-ak@see.eng. osaka-u.ac.jp; izumi@see.eng.osaka-u.ac.jp).

H. Okazaki is with Eishin Co., Ltd, Japan.

Color versions of one or more of the figures in this paper are available online at http://ieeexplore.ieee.org.

Digital Object Identifier 10.1109/TASC.2008.920830 the higher magnetic field of $1 \mathrm{~T}$, the magnet has not enough performance to separate particles because the strong magnetic field can be produced only in the vicinity of the magnet. The stronger magnetic force with larger area is necessary for the separation.

The superconducting magnet was used to generate high magnetic field gradients around the magnetic filter inserted into the boa. The martensitic SUS304 particles passing through the filter were separated by the strong magnetic force. A magnetic filter generates a strong magnetic field gradient and has been used for high gradient magnetic separation (HGMS) [2]-[6]. When the magnetic filter was used for powder separation, the blockade occurs in the filter due to the cohesion of the particles.

In this study, magnetic filter having square-shaped cross section was employed to avoid the blockage and to obtain high separation efficiency. In this work, the experiments and the calculations were made for designing the magnetic filters which can maintain the stable separation efficiency.

\section{THEORY OF MAGNETIC SEPARATION}

Magnetic force to a spherical particle $F_{M}$ is written as follows, where $r$ is radius of dispersed particle, $\mu_{0}$ is magnetic permeability in vacuum $\left(4 \pi \times 10^{-7} \mathrm{H} / \mathrm{m}\right), H$ is strength of external magnetic field, $\chi_{p}$ is magnetic susceptibility of ferromagnetic particle, and $\chi_{f}$ is that of dispersed particle.

$$
\begin{aligned}
F_{M} & =\frac{4}{3} \pi r^{3} \mu_{0} \frac{9\left(\chi_{p}-\chi_{f}\right)}{\left(3+\chi_{f}\right)\left(3+\chi_{p}\right)} \text { HgradH } \\
& \cong \frac{4}{3} \pi r^{3} M_{s} \text { gradH }
\end{aligned}
$$

The magnetic force $F_{M}$ is not always proportional to the external magnetic field $H$, because the magnetization $M\left(=\mu_{0} \chi_{p} H\right)$ of ferromagnetic materials saturates at comparatively low magnetic field. When the magnetization of the particle saturates, $F_{M}$ is proportional to the product of the saturated magnetization $M s$ and $\operatorname{gradH}$. In this case, high magnetic field gradient is required to obtain strong magnetic force. The $r$ and $M s$ in (1) are determined by the characteristics of the object.

In all experiments, the deformed martensitic SUS304 particles were chosen. Hereafter, the martensitic SUS304 particle is described as SUS304ma.

Fig. 1 show the magnetization curve of SUS304ma that separated in the experiments is evaluated by Quantum Design Magnetic Property Measurement System.

\section{EXPERIMENTAL}

\section{A. Design of Magnetic Separation System for Powder Mixture}

The photograph and the schematic diagram of the superconducting magnetic separation system are shown in Fig. 2. The 


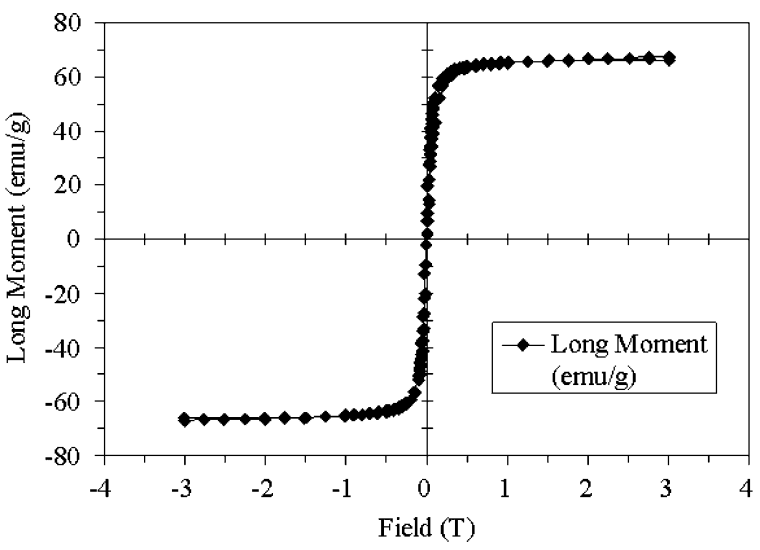

Fig. 1. Magnetization curve of SUS304ma. The horizontal axis shows the external magnetic field $\mathrm{B}$ and the vertical axis shows magnetization $\mathrm{M}$.
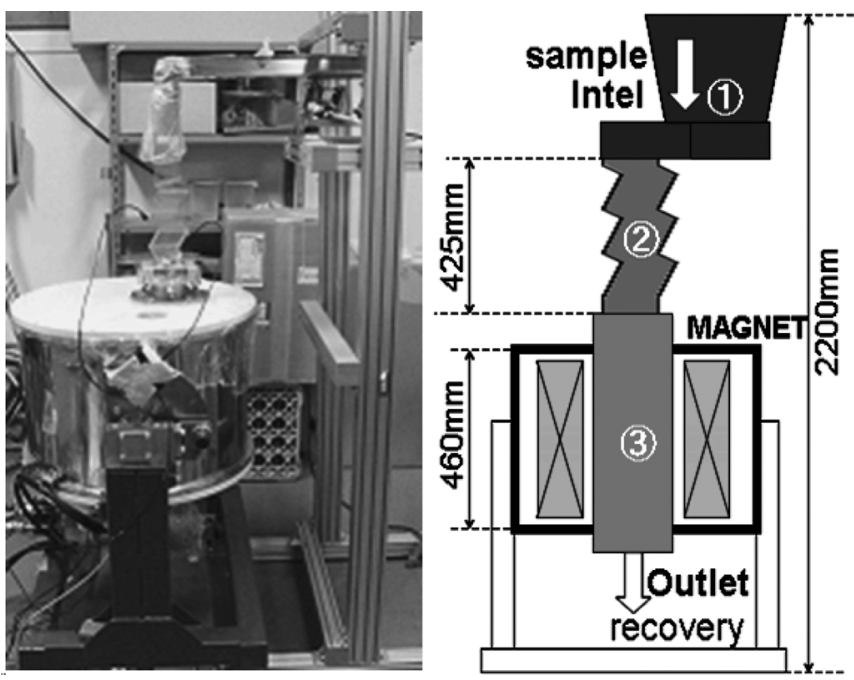

Fig. 2. Photograph and schematic diagram of the superconducting magnetic separation system. (1) feeder (2) dispersion unit (3) magnetic separation unit.

main unit of the system consists of three parts: (1) a feeder for stable supply of the sample to a dispersion unit, (2) a dispersion unit for dispersion of aggregated powder with air blow, and (3) a magnetic separation unit. The dispersion unit (2) is a stepwise vessel made of acrylate resin which connects (1) with (3). The air was provided by a compressor through a zeolite filter. The particles were dispersed to certain extent and decreased the aggregation. The cryogen-free superconducting magnet $(10 \mathrm{~T}$ of maximum magnetic field, JASTEC) was used for the separation. The boa diameter was $100 \mathrm{~mm}$ and the length was $460 \mathrm{~mm}$. Various magnetic filters were designed and inserted in the boa. By using the filter, high gradient magnetic separation (HGMS) was performed.

At first the magnetic separation using wire filters that could generate the high magnetic gradient and were often used for HGMS was studied. Magnetic wire filter with $1.0 \mathrm{~mm}$ in diameter and $5 \mathrm{~mm}$ in grid spacing were made of SUS430. Fig. 3(a) shows the photograph of a filter. The 32 stacks of the filters were installed in the magnet with intervals of $10 \mathrm{~mm}$. Because the position of the filter cannot be specified, the direction of the wire was arranged at random. The sample powder was made by mixing $0.05 \mathrm{~g}$ of martensitic SUS304 $(25 \mu \mathrm{m}$ in average particle diameter) with $50 \mathrm{~g}$ of silica particle $(2 \mu \mathrm{m}$ in average particle

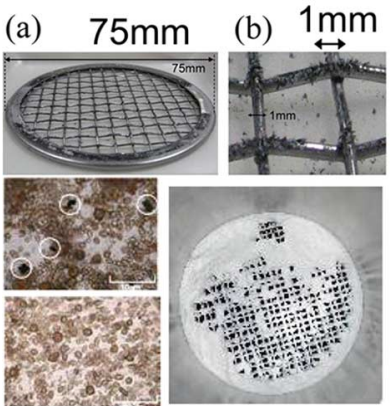

(c) (d)

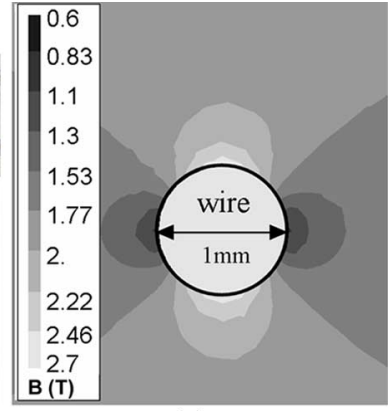

(e)
Fig. 3. Magnetic separation using wire filters (a) a wire filter, (b) sample after separation, (c) particles captured at the surface of wire filters, (d) blockaded magnetic filter, and (e) magnetic field around a wire of the filter.

diameter). The sample was put in the separator at $25 \mathrm{~g} / \mathrm{min}$ in feeding speed. The external magnetic field was changed from 0 to $2 \mathrm{~T}(0.25,0.5,1$, and $2 \mathrm{~T})$. Fig. 3(b) shows the photomicrograph of the sample after separation in 2 T. Fig. 3(c) shows the photomicrograph of the particles captured at the surface of the wire filter after degaussing. Though the separation efficiency could not be calculated only from the results of microscopic observation, SUS304ma particle was not found after the separation under the external field of $0.5,1$ and $2 \mathrm{~T}$. On the other hand, SUS304ma particles were found after the separation with $0.25 \mathrm{~T}$. Based on this result and the magnetization curve, $2 \mathrm{~T}$ was adopted for the external magnetic field in this experiment.

Magnetic field generated around the wire of the filter was calculated using ANSYS10.0 (Cybernet Co., Ltd.). In the model, the magnetic field of $2 \mathrm{~T}$ was applied at the center of the magnet boa (current density $2.2 \mathrm{E} 8 \mathrm{~A} / \mathrm{m}^{2}$ ). Seven stacks of magnetic filters were arranged in the area of $50 \mathrm{~mm}$ in length and $70 \mathrm{~mm}$ in width locating in the center of the magnet boa. Surface magnetic flux density and magnetic gradient around the magnetic filters were calculated. The result shows that magnetic flux density was $2.65 \mathrm{~T}$ and magnetic gradient was $2000 \mathrm{~T} / \mathrm{m}$ on the wire surface. The strong magnetic gradient was generated at the range of $0.5 \mathrm{~mm}$ from the wire surface (near 1000-2000 T/m). The SUS304ma was accumulated on the wire surface.

If the grid spacing is too wide, the window where the SUS304ma is not caught is formed and decreases the separation efficiency. In this experiment, the grid spacing was $5 \mathrm{~mm}$. In spite of the wide grid spacing, the filter showed good separation efficiency because the angle of the wires in filters was arranged at random, and there were little window. Though the particles were captured on filters, the filter comes to be obstructed over time. Fig. 3(d) shows the photograph of the state of obstruction.

The filter was obstructed even when the direction of the filter wire were unified. In being half lattice spacing, the filter was obstructed more quickly. The dry powder was aggregated easily due to the electrostatic force. In mesh filter, the obstruction happens easily because the spacing for passing through the powder is relatively small. When the spacing of the filter was expanded to avoid the obstruction, the separation efficiency would be decreased markedly. The magnetic filter should show no obstruction with aggregated powder and produce larger magnetic force. The both requirements are difficult to be satisfied by the wire filter. Therefore, the separation of the fine particle using magnetic wire filter is difficult. 


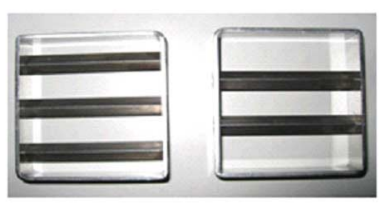

(a)
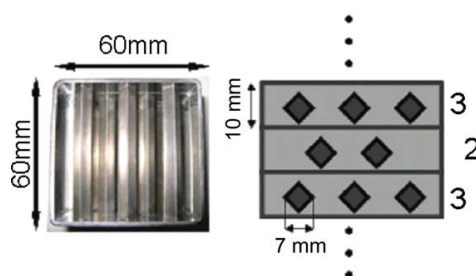

(b)

Fig. 4. Magnetic separation using magnetic filter with square cross section: (a) photograph of magnetic filters with steel rectangular column, (b) schematic illustration of the filter setting, and (c) the shape of three kinds of filter.

\section{B. Magnetic Filter With Square Cross Section 1}

In the experiment with the mesh filter, the powder came to be obstructed in the separator. In order to avoid the obstruction with the particles without decreasing magnetic force to separate SUS304ma particles, the filter with square cross section was designed. As shown in Fig. 4, two types of filters were fabricated. Filters with two or three steel rectangular column were fabricated Fig. 4(a).

The filters having two or three column were set in the magnet boa alternately and totally 12 layers of the filters were installed. The filters were installed so as not to form the areas where the particles pass through by directly. The shape of steel column were as follows: (i) column with square shaped cross section of $7 \mathrm{~mm}$ in diagonal length and 90 degrees in apex angle, (ii) column with rhombus shaped cross section of $7 \mathrm{~mm}$ or $12 \mathrm{~mm}$ in diagonal length and 60 degrees in upper and lower apex angle, (iii) column with water droplet shaped cross section of $60 \mathrm{de}-$ grees in upper apex angle. The magnetic field around the filters was analyzed by FEM, and then the experiments with those filters were performed based on the analysis.

Three kinds of filters mentioned above were used. $0.05 \mathrm{~g}$ of SUS304ma (average particle diameter: $25 \mu \mathrm{m}$ ) and $50 \mathrm{~g}$ of silica particles (average particle diameter: $2 \mu \mathrm{m}$ ) were mixed together $(0.1 \mathrm{wt} \%)$, and the mixture was used as a model sample. Magnetic separation was performed by applying $2 \mathrm{~T}$ of external magnetic field. Feeding speed was set at $25 \mathrm{~g} / \mathrm{min}$ which is common in all experiments. After magnetic separation, separated residue which passed through the magnetic filter was analyzed using XPS (X-ray Photoelectron Spectroscopy), and the content of SUS304ma that could not be removed was estimated. The separation efficiency was calculated from the initial content of SUS304ma in sample and the content after separation.

The results of magnetic field analysis around the magnetic filter when $2 \mathrm{~T}$ of external magnetic field was applied to the magnetic filters are shown in Fig. 5, (i) square shaped, 90 degrees filter, (ii) rhombus shaped, 60 degrees filter, (iii) water droplet shaped, 60 degrees filter. The characteristics of the magnetic field distribution of (i)-(iii) were discussed as followings, though they produce the smaller magnetic field and the smaller magnetic gradient than the magnetic thin wire (magnetic flux density: $2.65 \mathrm{~T}$, magnetic field gradient: $2000 \mathrm{~T} / \mathrm{m}$ ).

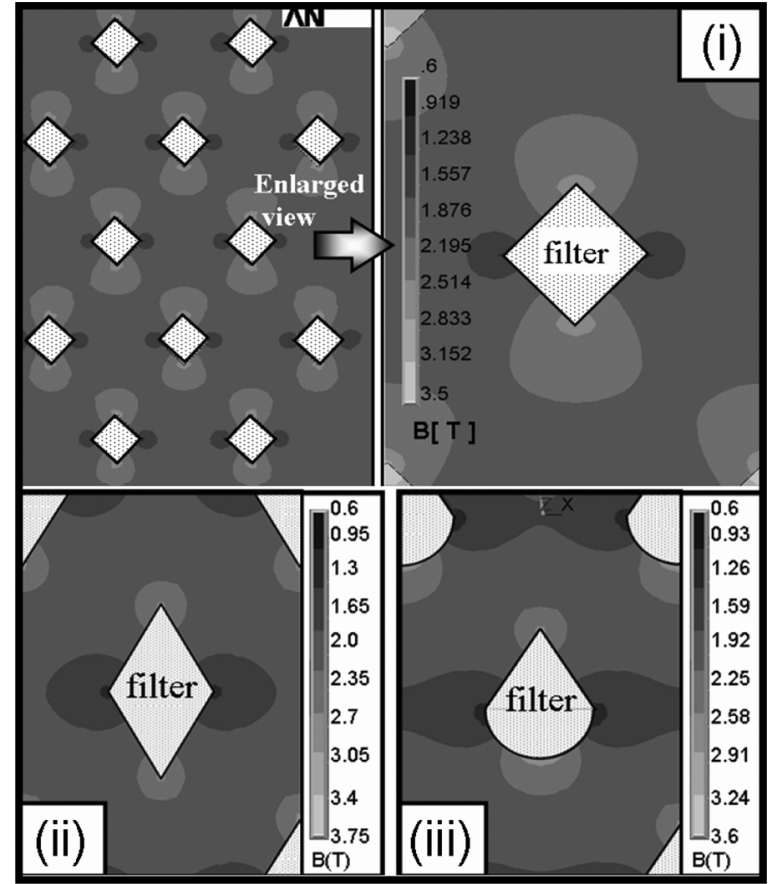

Fig. 5. Analysis of magnetic field around the magnetic filter.

(i) On the surface of upper and lower corner of the filter, magnetic flux density and magnetic gradient were as high as $2.8 \mathrm{~T}$ and $1100 \mathrm{~T} / \mathrm{m}$, and the high magnetic field region is expanded $4-5 \mathrm{~mm}$ in the vertical direction.

Even at the $0.5 \mathrm{~mm}$ distance from the surface, it has strong magnetic field gradient of $500 \mathrm{~T} / \mathrm{m}$. At the right and left corner a magnetic gradient is strong only in the vicinity of filter surface, and the force that prevents passage of particles from the top to the bottom of the stacks of the filters are not induced. SUS304ma was mainly trapped on the upper corner of the filter.

(ii) On the surfaces of the upper and lower corner of the filter, magnetic flux density is $3.2 \mathrm{~T}$ and magnetic gradient is $1600 \mathrm{~T} / \mathrm{m}$. Therefore magnetic flux density is higher than (i). Other characteristics are similar to (i).

(iii) On the surface of the upper corner, the magnetic gradient is $1700 \mathrm{~T} / \mathrm{m}$ which is $100 \mathrm{~T} / \mathrm{m}$ higher than (ii), and magnetic flux density is $3 \mathrm{~T}$. The magnetic gradient is 500 $\mathrm{T} / \mathrm{m}$ on the lower corner. Other characteristics are similar to (i).

Separation efficiency with filter (i) was $74 \%$, with filter (ii) $90 \%$ and with filter (iii) $94 \%$. The blockage has not taken place with magnetic separation with every stacked the filters.

Since the powder samples come down in a vertical direction, the powders are accumulated on the upper corner of filters by magnetic force. Samples are not accumulated on right and left corner because the relatively smaller magnetic forces are produced and hence the samples can pass through filters without blocking. Then, powdered SUS304ma escaped from the first filter is accumulated on the upper corner of next filter. This separation mechanism is repeated until the last filter and increases the separation efficiency.

There is a $20 \%$ difference in separation efficiency between (i) and (ii), (iii). The SUS304ma powder is aggregated with the 


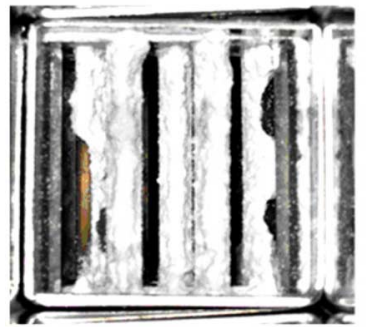

(i)

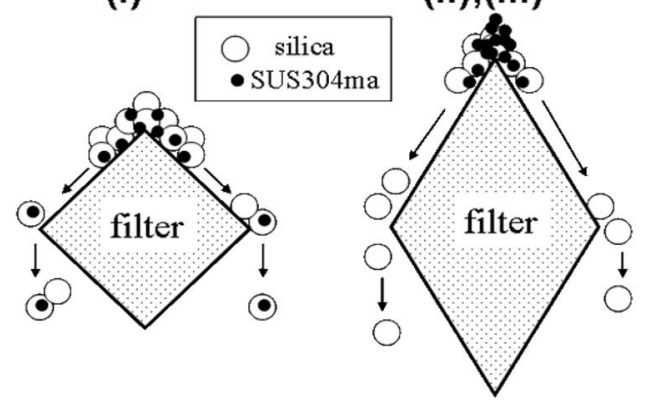

Fig. 6. Distribution of the particles accumulated on the filter.

silica powder on the upper corner of filter (i), and the excessive accumulated powder falls down because of its own weight. This may be the cause of decrease in separation efficiency. Though the collapse can be restrained by the stronger magnetic force, the strengthening of magnetic force only makes the time longer until the collapse. It is necessary to drop silica particle as fast as possible not to adhere on the aggregated blocks. It was shown that the decrease in separation efficiency is improved using the filter with sharper corner. Fig. 6 shows photos of the particles accumulated on the filter. The aggregated powder also piled up on the surface of filter (i). On the other hand the aggregated powder was not found on the surface of the filter (ii) and (iii) though the SUS304ma was accumulated on upper corners of the filters

\section{Magnetic Filter With Square Cross Section 2}

To investigate how much the aggregation of the particles influence the blockage of the filter and the dropping of trapped SUS304ma, experiments with higher density samples (SUS304ma content: 0.5 and $1.0 \mathrm{wt} \%$ ) using the filter (ii) and (iii), which had high separation efficiency in previous test, were performed. The results were described as follows. When the sample density was $0.5 \mathrm{wt} \%$, the separation efficiencies of the filter (ii) and (iii) were 94 and 92\%, respectively. When the sample density was $1.0 \mathrm{wt} \%$, the separation efficiencies of the filter (ii) and (iii) were 94 and 96\%, respectively. In both cases, the increase of SUS304ma content did not influence the separation efficiency of the filter. The results of all the experiments with iron multistage filters in this study are shown in Fig. 7. The separation efficiencies of the filter (ii) and (iii) were constantly high regardless of SUS304ma content in samples. It seems that in this experimental condition the magnetic force for trapping

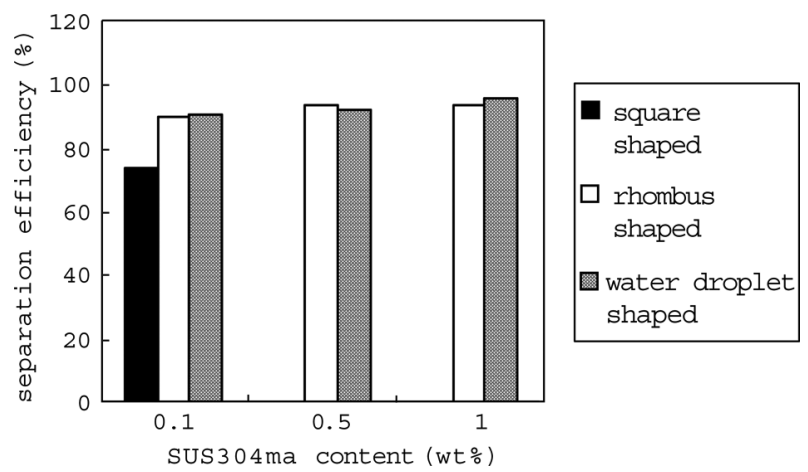

Fig. 7. Results of all the experiments with iron multistage filters.

the sample and the structure of the separator were optimized enough that the influences of the aggregation were negligible.

\section{CONCLUSION}

The dry type magnetic separation is significantly different from the wet type one because the medium of separation object is not liquid but gas. Magnetic separation of particle is difficult because fine particles are aggregated each other by the cohesion between them. Using the magnetic filter with square cross section, SUS304ma were separated on the corner of the filter without blockage. When the corner angle of a filter became sharper, the separation efficiency increased up to $20 \%$. The separation efficiency, more than $90 \%$, was achieved. We are planning to introduce the air flow into the separation unit to avoid the cohesion between particles. The magnetic field analysis suggests us the possibility of improvement. The advantage of our device is a structure of the system in which the filters can be exchanged according to the properties of the object. The next stage of this study is to introduce air flow into separator unit and to take a countermeasure against the static electricity by grounding the apparatus. The optimization of the magnetic separation system using actual samples is being planned.

\section{REFERENCES}

[1] F. Mishima, S. Yamazaki, K. Yoshida, H. Nakane, S. Yoshizawa, S Takeda, Y. Izumi, and S. Nishijima, "A study on the development of an open-gradient magnetic separator under dry condition," IEEE Trans. Appl. Supercond., vol. 14, no. 2, pp. 1561-1564, 2004.

[2] J. H. P. Watson, "Magnetic filtration," J. Appl. Phy., vol. 44, no. 9, pp. 4209-4213, September 1973.

[3] R. R. Birss, R. Gerber, and M. R. Parker, "Theory and design of axially ordered filters for high intensity magnetic separation," IEEE Trans. Magn., vol. 1, no. 6, pp. 892-894, Nov. 1976, MAG-12.

[4] S. Uchiyama, S. Kondo, and M. Takayasu, "Performance of parallel stream type magnetic filter for HGMS," IEEE Trans. Magn., vol. 1, no. 6, pp. 895-898, Nov. 1976, MAG-12.

[5] S. Nishijima, K. Takeda, K. Saito, T. Okada, S. Nakagawa, and M. Yoshiwa, "Applicability of superconducting magnet to high gradient magnetic separator,” IEEE Trans. Magn., pp. 573-576, 1987.

[6] Y. Kakihara, T. Fukunishi, S. Takeda, S. Nishijima, and A. Nakahira, "Superconducting high gradient magnetic separation for purification of wastewater from paper factory," IEEE Trans. Appl. Supercond., vol. 14, no. 2, pp. 1565-1567, 2004. 